\title{
Soluble Guanylate Cyclase Redox State and Usefulness of Nitric Oxide- Enhancing Drugs in Monocrotaline-Induced Pulmonary Hypertensive Rats
}

\author{
Masashi Tawa ${ }^{1,2}$, Takahide Furukawa ${ }^{2}$, Hiroko Tongu ${ }^{2}$, Mai Sugihara ${ }^{2}$, Satoko Taguwa ${ }^{2}$, \\ Misaki Yamanaka², Yoko Yano ${ }^{2}$, Hiroaki Matsumori ${ }^{2}$, Tatsuya Sawano ${ }^{2,3}$, Ryosuke Tanaka ${ }^{2}$, \\ Mamoru Ohkita ${ }^{2}$, Yasuo Matsumura ${ }^{2}$
}

\author{
${ }^{l}$ Department of Pharmacology, Kanazawa Medical University, Japan, ${ }^{2}$ Laboratory of Pathological and Molecular \\ Pharmacology, Osaka University of Pharmaceutical Sciences, Japan, ${ }^{3}$ Division of Molecular Pharmacology, \\ Department of Medicine, Tottori University, Japan
}

Background: The heme moiety of soluble guanylate cyclase ( $\mathrm{sGC}$ ), an important target of nitric oxide (NO), exists as a mixed configuration: the reduced, oxidized, and heme-free form. Since NO can activate only the first configuration, a change from the NO-sensitive reduced form to the NO-insensitive oxidized/heme-free form results in reduced NO bioavailability. This study was carried out to investigate whether disruption of the sGC redox equilibrium is seen in pulmonary hypertension $(\mathrm{PH})$ and whether treatment with NO-enhancing drugs can halt the disease progression.

Methods: Rats were injected subcutaneously with saline or $60 \mathrm{mg} / \mathrm{kg}$ monocrotaline (MCT). At 14 days after injection, vascular reactivity of isolated extralober pulmonary arteries to BAY 41-2272 (reduced sGC stimulant) and BAY 60-2770 (oxidized/heme-free sGC stimulant) was assessed by organ chamber technique. In another series, isosorbide mononitrate $(0.3$ or $1 \mathrm{~g} / \mathrm{L}$ ) or sodium nitrite $(30$ or $300 \mathrm{mg} / \mathrm{L}$ ) was administered in drinking water for the last 14 days (from day 15 to day 28 after MCT injection), and their therapeutic potential was evaluated.

Results: At 14 days after MCT injection, pulmonary arterial medial thickening was observed, whereas right ventricular systolic pressure (RVSP) elevation and right ventricular (RV) hypertrophy were still not developed. In addition, the sGC stimulator BAY 41-2272 and the sGC activator BAY 60-2770 both produced a relaxation of pulmonary arteries, which was comparable between saline- and MCT-injected rats. At 28 days after MCT injection, significant pulmonary arterial medial thickening, RVSP elevation, and RV hypertrophy were pronounced, the former two of which were prevented by treatment with isosorbide mononitrate. However, the beneficial effects on RVSP and pulmonary vascular remodeling were not seen when a high dose was administered. Exactly the same results were obtained in sodium nitrite treatment either. Interestingly, plasma nitrite/nitrate levels were not increased by these NO-enhancing drugs of the dose which provided more therapeutic advantages.

Conclusions: These findings suggest that the sGC redox state is not disrupted in the early stage of MCT-induced PH. Furthermore, therapy with an adequate amount of NO supply is proposed to be a useful option to prevent the progression of $\mathrm{PH}$. 